\title{
Performance evaluation of AODV, DSR and DSDV in mobile ad-hoc network using NS-2
}

\author{
Fan-Shuo KONG, Bei-Bei CUI \\ School of Software Engineering, Beijing University of Technology, Beijing, China \\ e-mail: kongfanshuo0224@163.com, cuibeibeibatty@163.com
}

\begin{abstract}
Mobile ad-hoc network (MANET) is a set of movable hosts established without existing network infrastructure and can be self-organized dynamically. MANET protocols have faced big challenges due to dynamic changing network topology and asymmetric network link. In this paper, we simulate AODV, DSDV, DSR routing protocols in network simulator NS-2 and evaluate and compare the performance metrics for each routing protocol using packet delivery ratio, average end to end delay of packets and normalized routing overhead. We conduct the simulation by varying the sending rate of source node ( 2 packets/s and 4 packets/s) with different pause time using node movement model and cbr source traffic model.
\end{abstract}

\section{Introduction}

Ad hoc networks consist of hosts without existing network infrastructure and can be self-organized dynamically. The idea of mobile ad hoc network is to maintain strong and selforganized function in mobile wireless networks by incorporating routing functionality into movable nodes[1]. A lot of work has been done to develop and improve routing protocols in ad hoc networks[2]. In MANET, the mobile host need to communicate with other mobile hosts which may not in its radio transmission range, so it is essential for routing protocols to finish these following functions, determine network topology, maintain network connectivity, schedule transmission, and route packet. Routing protocols in ad hoc network were designed according to the goals of dynamic topology maintenance, minimal control overhead, minimal process overhead, multi hop routing ability, and loop prevention[3]. According to the strategy of routing, the routing protocols in ad hoc networks is categorized as tabledriven(proactive) routing protocols and on-demand(reactive) routing protocols. The following figure 1 shows the classification of routing protocols. Each of these protocols performs differently in different wireless conditions. Hence it is necessary to analyze performance of these routing protocols to know its behavior and suitable working environment. Several factors may affect the overall performance of routing protocols in ad hoc network. For example, the link failures caused by node mobility will impact on packet transfer and quality of service (QoS). the internal characteristic of node itself like node speed or pause time, external factor like network size, traffic intensity will have a considerable impact on the overall performance of ad hoc network.

This paper is organized into five sections. The previous section covers the introduction of ad hoc network. Next section describes a detailed study on different types of MANET routing protocols. Section III describes the simulation methodology. Section IV describes performance metrics and result analysis, and the final section concludes the paper.

\section{Literature Survey}

\subsection{0n-Demand routing protocol}

On-Demand routing protocol first starts a process of path discovery and then transfers routing information when a path is required to get to the destination node.

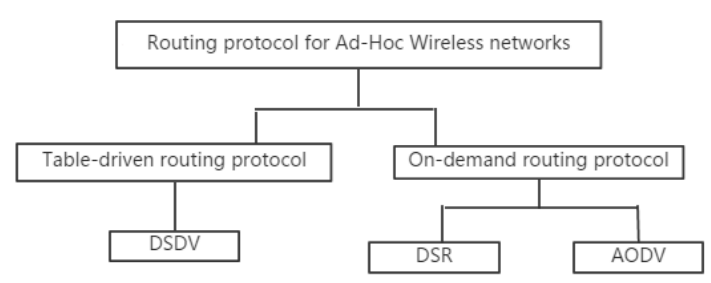

Fig. 1. Classification of routing protocols 


\subsubsection{DSR}

Dynamic Source Routing protocol is an on demand protocol, it overcomes the bandwidth consumption by removing periodic table update messages which is mandatory in tabledriven approach[4]. DSR has two phases, Route Discovery phase to find a path and Route Maintenance phase to maintain a path. When there is a need to transfer packets to a destination, the source node will query available route from source to destination in its route cache, if no route is available, it will initialize a process of route discovery[5]. The request packet(RREQ) sent by source node includes the destination address at first, and the series of intermediate nodes' address will be added to the request packet through the broadcast flooding. When destination node receives RREQ packet, it will send back a route reply(RREP) packet to source node through the reverse path included by RREQ packet. After the establishment of route discovery, the data packet sent by the source carries complete ordered series of nodes which the packet must pass in packet's header, this could prevent the loop and eliminate the need of updating route information in the intermediate nodes which the packet is forwarded through. In route maintenance phase, once the link is broken between nodes, route error messages will be sent to all nodes in the network. Intermediate node use cache to maintain several routes to destination node, so sometimes if intermediate node gets the RREQ packet, it directly sends the path information to source node ${ }^{[5]}$.

\subsubsection{AODV}

Ad-hoc On-demand Distance Vector protocol is on-demand routing protocol[6]. When the source wants to transfer data, it will try to establish a path between itself and the destination. When a node wants to build a traffic connection, it first broadcast a request(RREQ) including the information of destination. The intermediate nodes process the RREQ, they record the reverse path to the source node in their route table, and then decide whether to broadcast the RREQ or not. When the node receives such a message, if it is the destination node itself or there is a route to the destination node in its route entry, it will send a reply(RREP) using the reverse route to the source node. The intermediate node will also record the reverse path to the destination when receiving the RREP. The source nodes then begin the process of sending data using the route entry which has the freshest sequence number of destination and minimum number of hops. The entries in the routing tables will be recycled in a regular interval. When the links is broken, the routing error packet(RERR) is flooded to the source node, and the process of route discovery restarts. Following figure 2,3 shows that how route will be established in AODV.

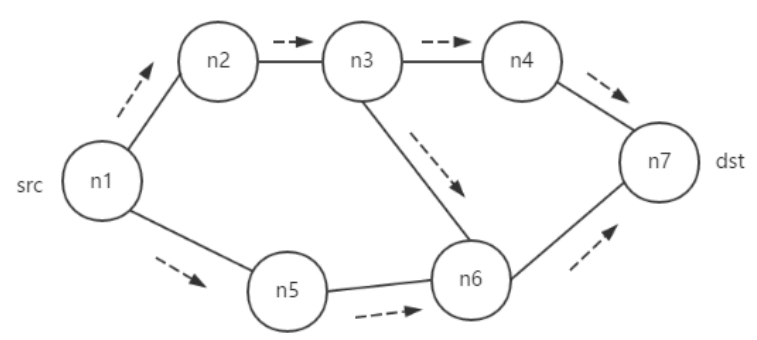

Fig 4. Route request broadcasting in AODV protocol

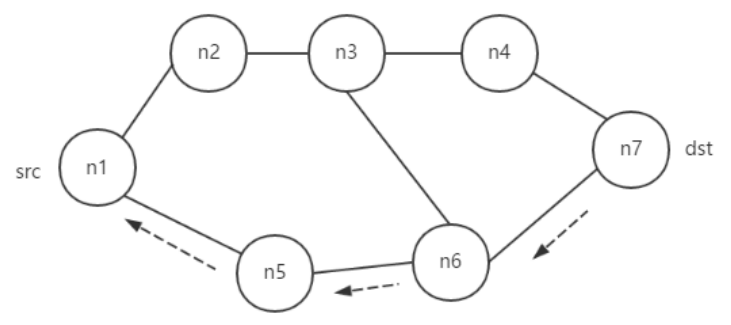

Fig 5. Route reply in AODV protocol

The main difference between DSR and AODV is in DSR, it carries complete ordered sequence of node path to be traversed in each data packet, while in AODV the source node and all intermediate nodes stores next hop information of each flow. Besides, DSR doesn't have beacon packets to tell neighbors of its existence, while in AODV, it requires periodic hello packet (beacon) transmission, which is used to inform its neighbors of its presence.

\subsection{Table-Driven routing protocol}

Table-Driven routing protocols maintain a table about topology information of whole network in each node just like wired network routing protocol. In order to maintain accuracy and consistency of route table, nodes will update all tables frequently.

\subsubsection{DSDV}

Destination Sequenced Distance Vector[7], is hop-by-hop distance vector routing protocol which requires each node to send routing updates in a regular interval. It is based on principles of classical Bellman-Ford Routing algorithm[8]. The routing table maintained by each node consists of records of the next hop for every reachable destination, the number of hops to destination and the sequence number assigned by destination. The sequence number is used to judge the freshness of route entry. If any change has occurred in its routing table from any received update, it will transmit its routing table update. The update is based on time-driven and event-driven, and "Full-dump" update or "incremental" update will be used to send the routing table updates. 


\section{Simulation Methodology}

\subsection{Simulation Modeling}

Simulation is the commonly used way to analyze the performance and behavior of wireless network before putting it in use in real application. Several network simulators can be used to simulate the scenario as accurate as in real time implementation. In this work, we used the discrete-event simulator NS2 (version 2.35) [9] to do the simulation, and conduct the performance analysis using AWK script[10]. we adopted several models integrated in NS2 simulator. We use IEEE 802.11 MAC model for MAC layer functionality. We adopt two Ray Ground propagation model for wireless signal propagation. For node mobility model, we use Random Waypoint Mobility model to implement dynamic network topologies. We use Radio Model for radio characteristics used by node with proper bandwidth and frequency. For Traffic Model, we use CBR, UDP Model for nodes to send traffic to destinations.

\subsection{Simulation Methods}

The aim of our experiments is to quantify the effects of various factors and their impact on the overall performance in ad hoc networks. Each test of the simulation accepts a mobile scenario file and a traffic scenario file as input. The mobile scenario is created by a transcript program called cbrgen.tcl, which describes specific movement of each node with exact time the motion occurs or direction changes. The traffic scenario is created by the tool setdest, which describes the exact connection pairs initialized by particular nodes with exact time connection begins. To evaluate the performance of a specific factor, we take 5 runs of simulation to generate 5 different scenario patterns and the average of these 5 outputs is calculated to be the performance of the considered factor. For each factor, we adopted 7 sample points to compare three different ad hoc protocols, DSR, DSDV, AODV. Hence, 105 runs of simulation are conducted to analyze each factor for three protocols. Network layer characteristics is the factors to be observed, so only changes in the strategy of routing is observed and varied, while other characteristics of physical layer(ground propagation model), link layer(MAC 802.11), transport layer(UDP), application layer(CBR) stay fixed.

\subsection{Simulation Parameters}

Simulation environment is in a $1000 * 300$ square with total 50 mobile nodes with constant maximum speed $20 \mathrm{~m} / \mathrm{s}$. We set traffic pattern with maximum 20 UDP connections between mobile nodes. The mobility of all nodes is done with various pause times from 0 to $900 \mathrm{~s}$. The packets sending rate of each source varies from 2 pkts/s to $4 \mathrm{pkts} / \mathrm{s}$. Simulation time is $900 \mathrm{~s}$. Detailed parameters are shown in table I.
TABLE I. SIMULATION PARAMETERS

\begin{tabular}{|l|l|}
\hline \multicolumn{1}{|c|}{ Component } & \multicolumn{1}{c|}{ Type } \\
\hline Channel Type & Channel/Wireless Channel \\
\hline Antenna Model & Omni Antenna \\
\hline $\begin{array}{l}\text { Radio Propagation } \\
\text { Model }\end{array}$ & Two Ray Ground \\
\hline Network Interface Type & Phy/ Wireless Phy \\
\hline Mac Layer Protocol & IEEE 802.11 \\
\hline Interface Queue Type & Queue/DropTail/PriQueue \\
\hline Number of Nodes & 50 \\
\hline Pause Times & $0,150,300,450,600,750$, \\
& 900 \\
\hline Number of Sources & 50 \\
\hline Topology Size & $1000 \mathrm{~m}$ x 300m \\
\hline Traffic Type & Constant Bit Rate (CBR) \\
\hline Packet Size & 512 Byte \\
\hline Packet send rate & $2 \mathrm{pkts} / \mathrm{s}, 4 \mathrm{pkts} / \mathrm{s}$ \\
\hline Simulation Time & $900 \mathrm{~seconds}$ \\
\hline Simulation Tool & NS-2.35 \\
\hline
\end{tabular}

\section{Performance metrics and RESULT ANALYSIS}

\subsection{Performance Metrics}

The performance metrics reflects the Quality of Service (QoS) of the wireless network. The following three performance metrics are considered to characterize the performance and behavior of DSR, DSDV, AODV routing protocols: Packet delivery ratio (PDR), Average end-to-end delay of data packets and normalized routing overhead.

\subsubsection{Packet delivery Ratio}

Packet Delivery Ratio (PDR) [11] measures the successful rate of packet delivery in ad hoc routing protocols. It is calculated that the total number of packets received by traffic destination divided by the total number of packets sent by traffic source.

$$
\text { packet_delivery_ratio }=\frac{\sum C B R \_ \text {received }}{\sum C B R_{-} \text {sent }}
$$

where, CBR_received is the total number of CBR packets received from destinations, $C B R_{-}$sent is the total number of CBR packets sent by sources.

\subsubsection{Average end-to-end delay of data packets}

Average end-to-end delay[12] of data packets is defined as the average delay of received data packets within a network. Endto-end delay mainly includes propagation delay, transmission delay, queuing delay and processing delay. It is calculated that 
the sum of delay which cumulated by every successfullyarrived data packets divided by the total number of received data packets.

average_end_to_end_delay $=\frac{1}{n} \sum_{i=1}^{n}\left(R T_{i}-S T_{i}\right)$

where, $\mathrm{n}$ is the total number of received packets, $R T_{i}$ is the received time of number i packet, $S T_{i}$ is the sent time of number i packet, $\mathrm{i}$ varies from 1 to $\mathrm{n}$.

\subsubsection{Normalized routing overhead}

Normalized routing overhead[13] is defined as how many control packets consumed by one successfully-arrived data packet. It is calculated that the total number of control packets divided by the total number of received data packets.

3)

normalize route overhead $=\frac{\sum \text { control_packet_sent }}{\sum \text { data_packet_received }}(4-$

where,control_packet_sent is the total number of control packets sent or forwarded by the nodes. data_packet_received is the total number of data packets received from destinations.

\subsection{Result Analysis}

\subsubsection{Packet Delivery Ratio:}

Graphs in figure 4,5 shows packet delivery ratio of combination of DSR, AODV and DSDV. It can be seen when communication send rate is 2.0 or 4.0 packets/s, AODV and DSR routing protocol packet delivery ratio showed similarly satisfied results. No matter of the node is under the condition of weak mobility(low pause time), or strong mobility(strong pause time), the packet delivery rate is over $95 \%$. However, packet delivery ratio of DSDV appears larger fluctuation with the change of the pause time, when the pause time equals to 0 s, DSDV protocol packet delivery rate is lower than $80 \%$. In general, In a short pause time(strong mobility), the performance of DSDV protocol is far less than AODV protocol and the DSR protocol.

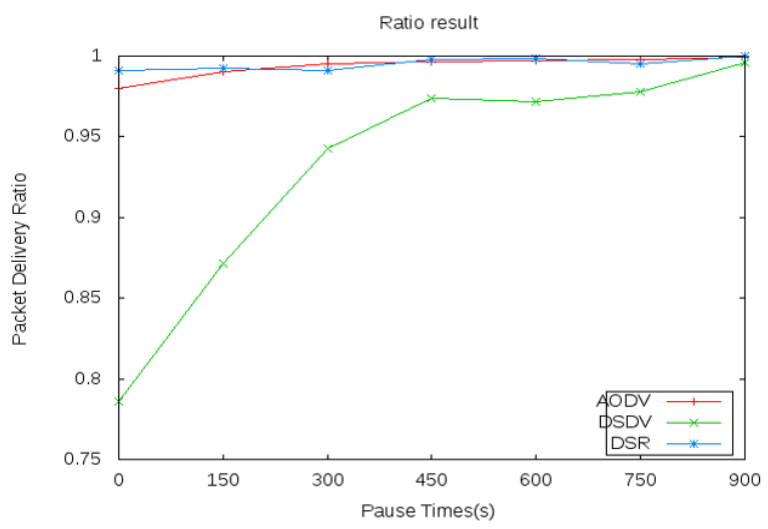

Fig.4. Packet Delivery Ratio of send rate 2 packets/s

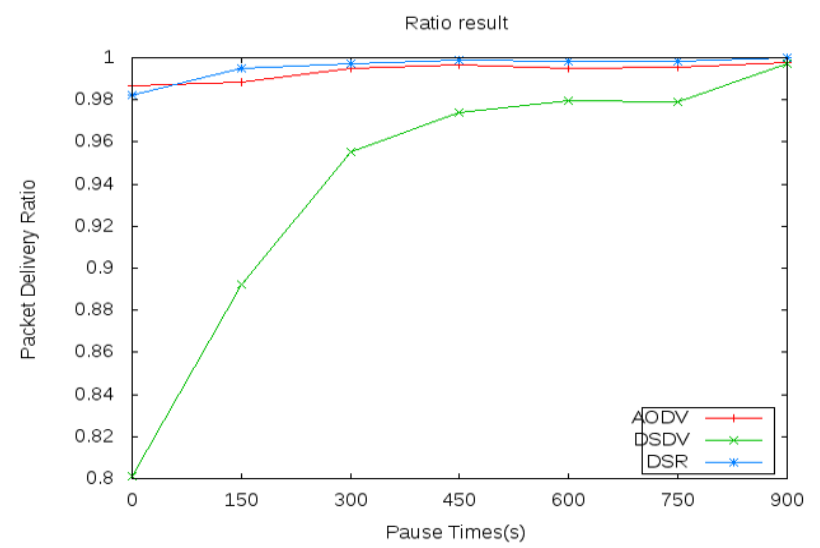

Fig.5. Packet Delivery Ratio of send rate 4 packets/s

\subsubsection{Average end-to-end delay of data packets:}

As graphs shown in figure 6,7, it is observed that at all sending rate, the end-to-end delay gradually decreases for all protocols as node mobility becomes lower (pause time is longer). When the node mobility is frequent, AODV performs much better than DSR and DSDV.

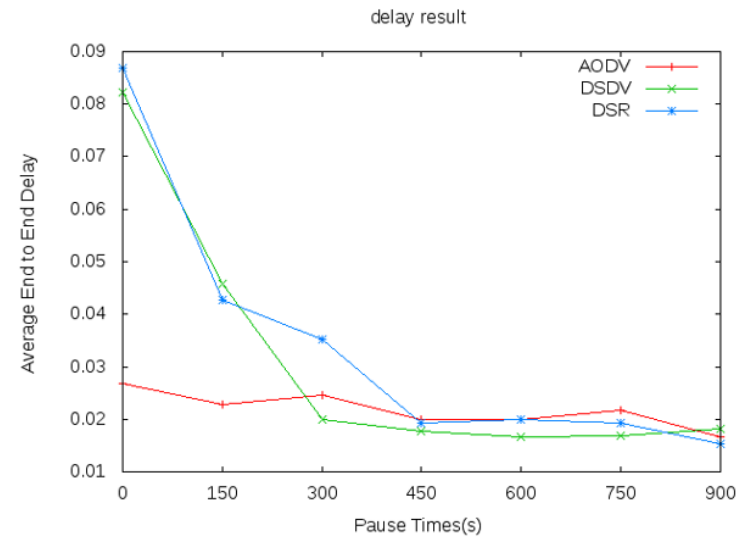

Fig.6. Average end-to-end delay of send rate 2 packets/s

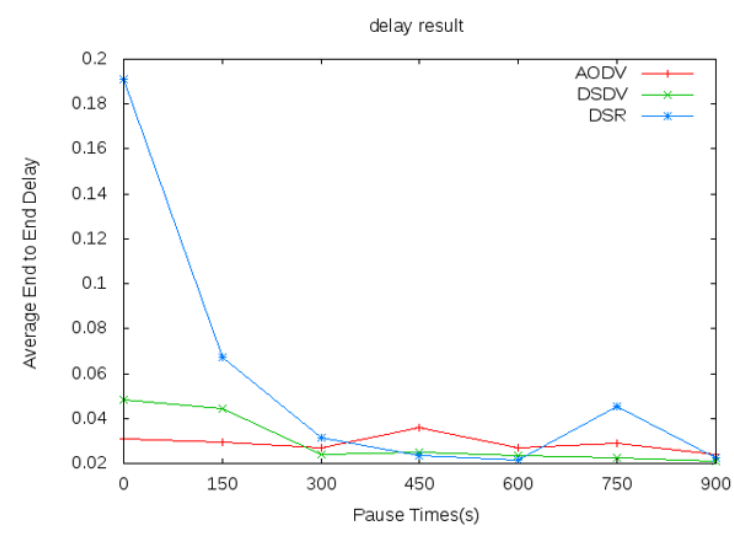

Fig.7. Average end-to-end delay of send rate 4 packets/s 


\subsubsection{Normalized routing overhead:}

Normalized routing overhead of DSDV protocol basically goes smoothly with the change of pause time and far more than that of AODV and DSR, that is because DSDV belongs to table-driven routing protocol, basically the routing overhead depicts small changes caused by network load and node mobility. While the AODV protocol and the DSR protocol is an on-demand routing protocol, the lower the mobility, the lower the normalized routing overhead is. Besides, in the same scenario, normalized routing overhead performance of DSR is always better than the AODV protocol, this is because the AODV protocol preserves only one routing entry for each destination node, when the link break, it will trigger a new round of routing discovery process. In general, DSR performs the best results than AODV and DSDV.

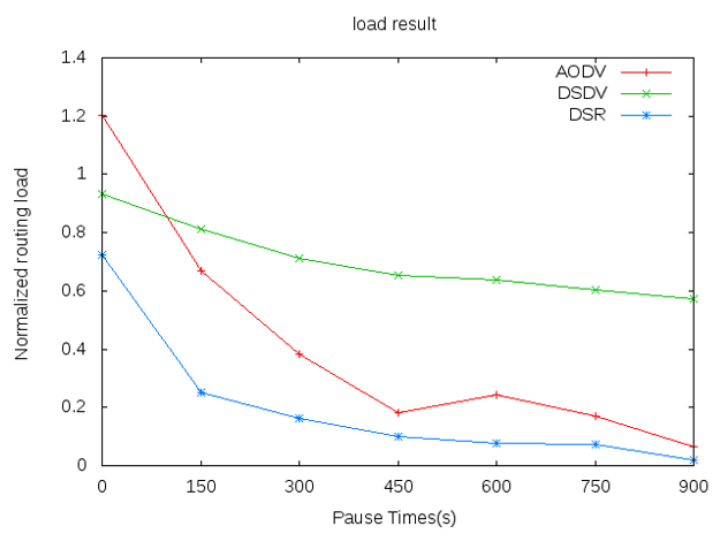

Fig. 8. Normalized Routing overhead of send rate 2 packets/s

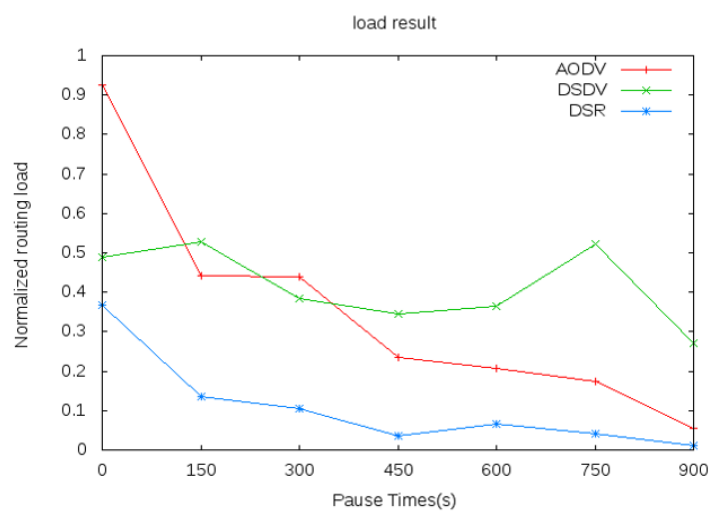

Fig. 9. Normalized Routing overhead of send rate 4 packets/s

\section{Conclusion}

This paper proposes an performance evaluation of three different routing protocols named as DSR, AODV, and DSDV. The process of the evaluation includes simulation of wireless network on platform in NS2. We analyzed them using three performance metrics named Packet Delivery Ratio, average end-to-end delay of data packets, and normalized routing overhead. From the simulation of these three routing protocols, we get the result as follows. For the result of packet delivery, DSR and AODV get the similarly better packet delivery ratio than DSDV. AODV get better performance in average delay than DSR and DSDV. DSR have minimum routing overhead than AODV and DSDV.

\section{References}

[1] Tonguz $\mathrm{O}$ and Ferrari G., "Adhoc Wireless Networks-A Communication -Theoritic Perspective, Wiley and Sons", 2009.

[2] Sarkar S, Basavaraju T.G. and Puttamadappa C., “ Ad Hoc Mobile Wireless Networks: Principles, protocols and applications", Auerbach Publications, 2008.

[3] Royer E.M.and Toh C., "A review of current routing protocols for adhoc mobile wireless networks", IEEE personal communications, 1999, pp. 46-56.

[4] D. B. Johnson and D. A. Maltz, "DSR : The Dynamic Source Routing Protocol for Multi-Hop Wireless Ad Hoc Networks," pp. 1-25.

[5] C. E. Perkins and E. M. Royer, "Ad Hoc On-Demand Distance Vector Routing," Proc. 2nd IEEE Wksp. Mobile Comp. Sys. and Apps., Feb. 1999, pp. 90-100.

[6] Perkins and Bhagwat "Highly Dynamic Destination Sequenced Distance-vector Routing (DSDV) for Mobile Computers, ACM SIGCOMM '94 Conference.

[7] The network simulator - ns-2. http://www.isi.edu/nsnam/ns/.

[8] Ould-Khaoua Ahmed and Al-Maashri. "Performance Analysis of MANET Routing Protocols in the Presence of Self-Similar Traffic". IEEE, ISSN- 0742-1303, First published in Proc. of the 31 st IEEE Conference on Local Computer Networks, 2006.

[9] Performance Measurement of Various Routing Protocols in Ad-hoc Network, Rahman, Islam, Alex, International Conference of Engineers and Computer Scientists 2009, IMECS 2009, Hong Kong.

[10] Fasee Ullah, Muhammad Amin and Hamid ul Ghaffar,"Simulating AODV and DSDV For Adynamic Wireless Sensor Networks" IJCSNS International Journal of Computer Science and Network Security, VOL.10 No.7,July 2010

[11] Asma Tuteja , Rajneesh Gujrl and Sunil Thalia,"Comparative Performance Analysis of DSDV, AODV and DSR Routing Protocols in MANET using NS2",International Conference on Advances in Computer Engineering, 2010.

[12] Sapna S. Kaushik1 \& P.R.Deshmukh2, "Comparison of effectiveness of AODV, DSDV and DSR routing protocols in Mobile Ad-Hoc Network" International Journal of Information Technology and Knowledge Management, Volume 2, No. 2, July-December 2009.

[13] S.Mohapatra, P.Kanungo , "Performance analysis of AODV, DSR, OLSR and DSDV Routing Protocols using NS2 Simulator," International Conference on Communication Technology and System Design 2011 ,SciVerse Science Direct, Procedia Engineering 30 (2012), pp69-76, doi:10.1016/j.proeng.2012.01.835.0 